\title{
Dipeptide derived from benzylcystine forms unbranched nanotubes in aqueous solution
}

\author{
Biswadip Banerji ${ }^{1 *}$, Sumit Kumar Pramanik ${ }^{1}$, Uttam Pal ${ }^{2}$ and Nakul Chandra Maiti ${ }^{2}$
}

\begin{abstract}
The essence of modern nanotechnology is manifested in the formation of well-ordered nanostructures by a process of self-association. Peptides are among the most useful building blocks for organic bionanostructures such as nanotubes, nanospheres, nanotapes, nanofibrils, and other different ordered structures at the nanoscale. Peptides are biocompatible, chemically diverse, and much more stable and can be readily synthesized on a large scale. Also, they have diverse application in biosensors, tissue engineering, drug delivery, etc. Here, we report a short cystine-based dipeptide, which spontaneously self-associates to form straight, unbranched nanotubes. Such self-assembled nanobiomaterials provide a novel possibility of designing new functional biomaterials with potential applications in nanobiotechnology. The formation of nanotubes in solution state has been demonstrated by atomic force microscopy and scanning electron microscopy. Infrared absorption and circular dichroism demonstrated the intermolecular $\beta$-sheet-like backbone hydrogen bonding in juxtaposing and stacking of aromatic side chains.
\end{abstract}

Keywords: Nanotube, Dipeptide, $\pi-\pi$ stacking, Cystine, Self-assembly

\section{Background}

There has been rapid advancement in the development of self-assembled nanobiomaterials, such as nanotubes, nanocrystals, and nanowires, which have potential application in electronics, biosensors, catalysis, drug delivery, and tissue engineering [1-4]. The physical and chemical properties of these nanomaterials are tunable by controlling their shapes and sizes [5]. There are various designing rules of the synthesis of these biomaterials, where secondary structure of the self-assembled fiber, the thickness of fiber, and hydrogel porosity, and different mechanical properties can all be varied predictably simply by changing the amino acid sequence, its concentration, the surrounding media, and its processing route [6-8]. Over the years, various natural selfassembling systems have served as inspiration for the design of novel building blocks on the nanoscale [9-11]. It has been already reported that cyclic peptides, amphiphilic peptides, and amyloid-inspired peptides can form ordered nanostructures with different morphologies including nanowires, nanotubes, nanovesicles, nanofibrils, and nanosheets [12-14]. The formation of these nanostructures

\footnotetext{
* Correspondence: biswadip.banerji@gmail.com

'Department of Chemistry, CSIR-Indian Institute of Chemical Biology, 4, Raja S.C. Mullick Road, Kolkata 700032, India

Full list of author information is available at the end of the article
}

is facilitated by the combination of different non-covalent forces that include electrostatic interactions, hydrogen bonds, hydrophobic interactions, and aromatic stacking interactions $[9,13,15]$. Unlike inorganic and carbon-based nanomaterials, the peptides undergo self-assembly process at ambient temperature and atmospheric pressure [16]. Besides, the peptides can be produced in large scale by simpler experimental methods. The self-assembled peptide nanostructures can further organize to form nanoscale devices [17]. Therefore, the application of self-assembling synthetic peptides as the building blocks of nanoscale devices is practical, robust, and affordable [18-20].

In this report, we present a novel peptide that, in solution, readily undergoes the formation of a linear nanotube with $\beta$-sheet-like backbone hydrogen-bonding pattern. The dipeptide constitutes two-side-chain benzyl-protected cystine moieties. This is possibly the first report for the formation of a cystine-based nanotube. First, this dipeptide has been synthesized, purified, and characterized. The $\beta$-sheet-like backbone arrangement of the peptide in the solid state has been confirmed by Fourier transform infrared (FT-IR) spectroscopy. The computational model calculation and density functional theory (DFT) study also support these data. 


\section{Results and discussions}

\section{Synthesis of the dipeptide}

The dipeptide was synthesized by conventional solutionphase methodology (Scheme 1). The $\mathrm{N}$-terminus was protected by the Bocgroup, and the $\mathrm{C}$-terminus was protected as methyl ester. Couplings were mediated using 1-ethyl-3-(3-dimethyllaminopropyl) carbodiimide hydrochloride/hydroxybenzotriazole (EDC. $\mathrm{HCl} / \mathrm{HOBt}$ ). The deprotection of methyl ester leading to the acid was performed by saponification (aq. $\mathrm{LiOH}$ ) method, and the Boc group was removed using $4 \mathrm{M} \mathrm{HCl}$ in 1,4-dioxane. The final tetrapeptide was fully characterized by ${ }^{1} \mathrm{H}-\mathrm{NMR}$, HRMS, and infrared (IR) spectroscopy.

\section{FT-IR study}

FT-IR spectroscopy studies were performed to determine the secondary structures of the peptide (Figure 1). In the case of $\beta$-sheet structure, the FT-IR spectra show two amide I transitions, one is a strong transition between 1,610 and $1,640 \mathrm{~cm}^{-1}$ and the other one is a weak transition between 1,680 and $1,700 \mathrm{~cm}^{-1}$. Here, the FT-IR spectra of the peptide show characterized $\mathrm{CO}$ stretching bands at around 1,631 and $1,680 \mathrm{~cm}^{-1}$ and the $\mathrm{NH}$ stretching band at around $3,304 \mathrm{~cm}^{-1}$, typical for intermolecularly

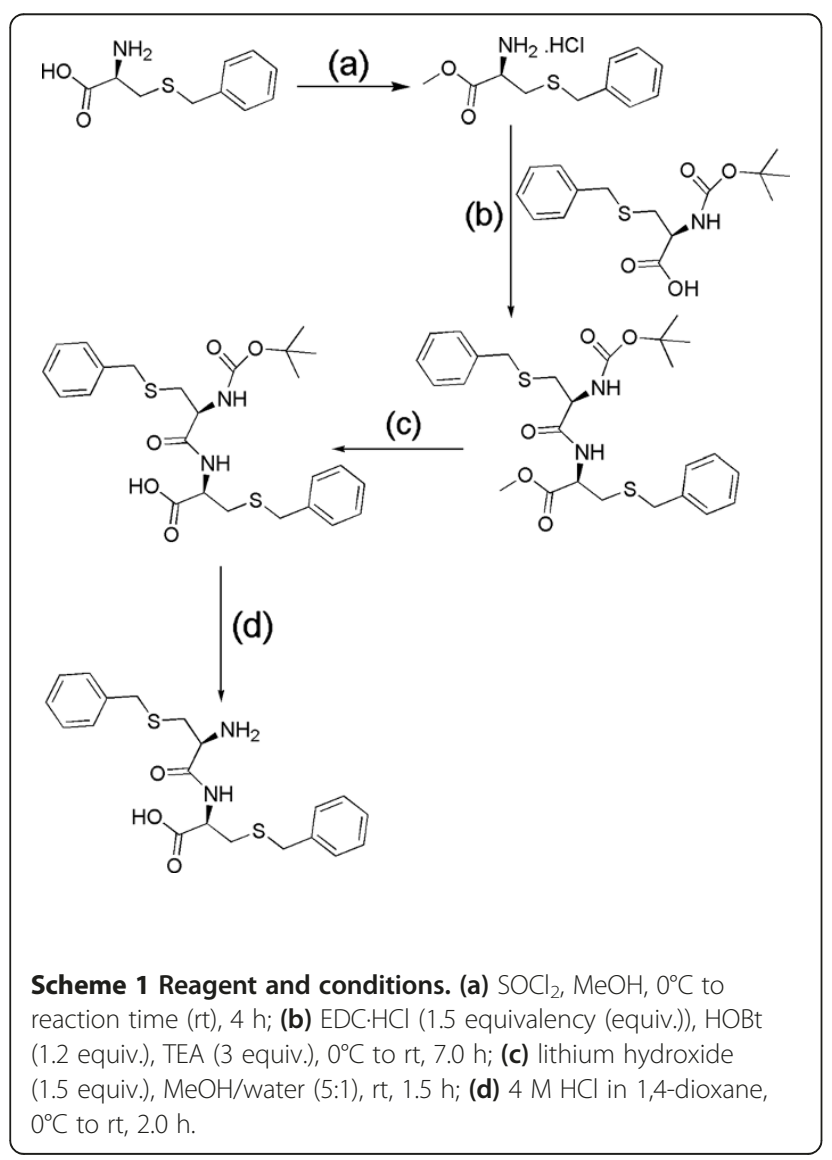

hydrogen-bonded $\beta$-sheet structures in solid. Furthermore, the $\mathrm{NH}$ bending frequencies of this dipeptide appear at $1,512 \mathrm{~cm}^{-1}$, suggesting the formation of a $\beta$-sheet structure, whereas absorption in the range 1,610 to $1630 \mathrm{~cm}^{-1}$, which is a characteristic of aggregated amyloid-like peptides, was not observed. IR frequencies were calculated using DFT level of theory. $\mathrm{NH}$ and COstretching frequencies were found to be 1,635 and $1,681 \mathrm{~cm}^{-1}$. It nicely corroborates with the experimentally obtained data.

\section{SEM and AFM imaging}

The scanning electron microscopy (SEM) images of the dipeptide (Figure 2A) show that it forms nanofibrils with a diameter of $80 \mathrm{~nm}$ and length of $30 \mu \mathrm{m}$. They are almost uniform in nature. The atomic force microscopy (AFM) image of the dipeptide also confirmed the formation of nanotubes with a diameter nearly $80 \mathrm{~nm}$ and length of $30 \mu \mathrm{m}$.

\section{Model of self-assembly process}

The dipeptide showed intermolecular $\beta$-sheet-like backbone hydrogen bonding in juxtaposing and stacking of aromatic benzyl side chains (Figure 3). They have a hydrophobic head with an array of overlapping benzyl groups similar to what is found in silk fibroin or spider silk assemblies [21]. The benzyl groups form packed hydrophobic interactions in water, which can be disrupted mechanically during sonication. However, these hydrophobic cohesive ends can find each other quickly in water since the exposure of hydrophobic alanine arrays to water is energetically unfavorable. Since the hydrophobic alanines' interaction is non-specific, they can slide diffuse along the nanostructure, like trains sliding along the rails [21]. Such fragments could form various assemblies readily through hydrophobic and intermolecular hydrogen-

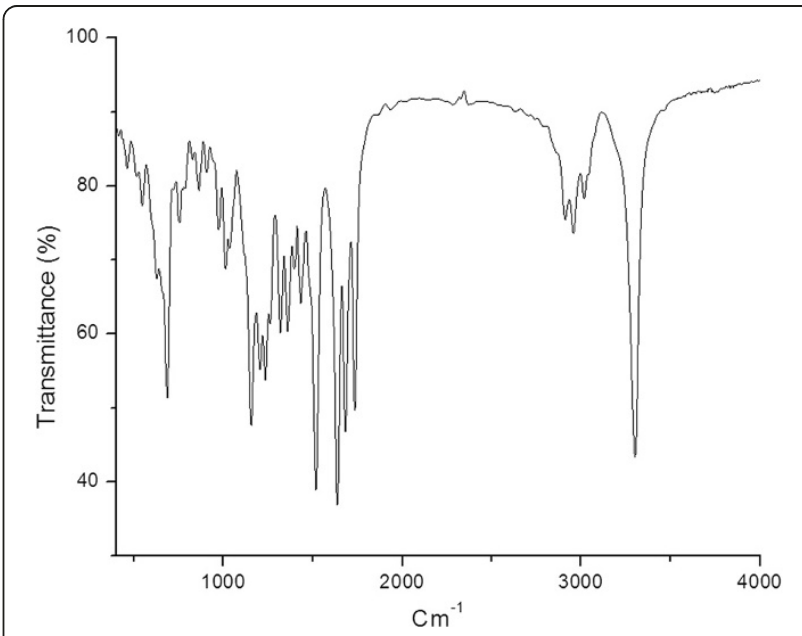

Figure 1 FT-IR spectra of the benzyl-protected cystine dipeptide. 


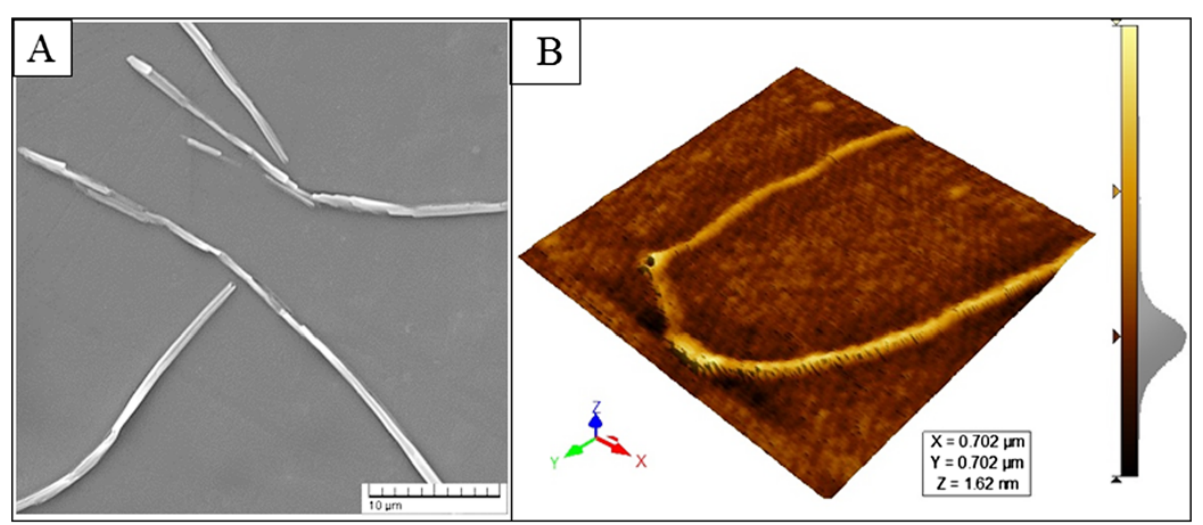

Figure 2 SEM image (A) and AFM image (B) of the dipeptide.

bonding interactions. The model of self-assembly process is shown in Figure 4.

Here, we have shown a model for plausible intermolecular interactions where the benzene ring of the benzyl groups shows $\pi-\pi$ stacking interaction with the benzene ring of the benzyl group of another dipeptide. The hydrogen bonding occurs between the $\mathrm{N}-\mathrm{H}$ of the amide group and the oxygen atom of the backbone carbonyl. The importance of hydrogen bonds and $\pi-\pi$ stacking interaction in the formation of nanotubes has been recognized and emphasized in the literature. It was pointed out that the formation of the hydrogen bonds is the necessary controlling factor in the formation of cylindrical shape. In this present system, the assembled phenyl rings could generate the possibilities of $\pi-\pi$ stacking interaction between the nearby dipeptide, which leads to the circular ring of the self-assemblies, though no $\beta$-sheet structure formed in solution phase. These two forces help form the nanotubes.

\section{CD spectroscopy}

The solution-state conformation of the peptide was also defined by circular dichroism (CD) spectroscopy. Circular dichroism revealed an extended conformation. The CD spectra of the dipeptide in water are shown in Figure 5. The peptide in dilute conditions showed a negative $C D$ band at $201 \mathrm{~nm}$. However, with an increase in peptide concentration, the CD absorption band red-shifted (approximately $3 \mathrm{~nm}$ ) to longer wavelengths, which indicates that at a higher concentration of the peptide due to the formation of oligomer, the amide geometry may be changed.

\section{Ultraviolet spectroscopy}

Two distinct absorption peaks in Figure 6 were observed due to the benzyl groups present in the dipeptide molecule: one at $260 \mathrm{~nm}$ and the other at $267 \mathrm{~nm}$. Scattering was also observed which indicates self-aggregation and higher oligomer formation. With the increasing concentration, scattering was also found to be increased.

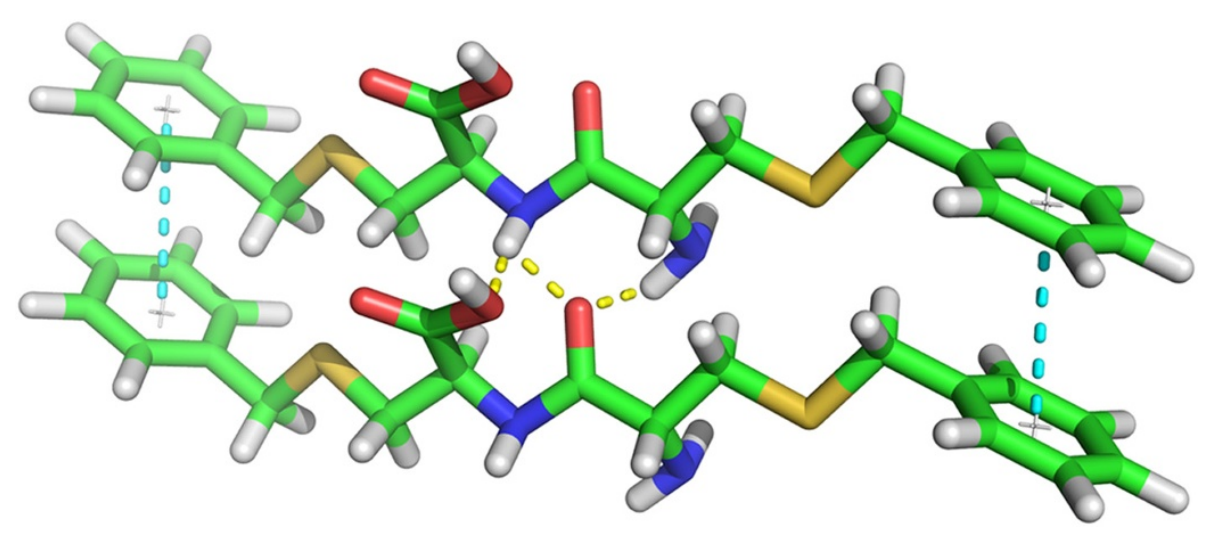

Figure 3 Plausible intermolecular interaction pattern. Illustrating the role of intermolecular $\beta$-sheet-like backbone hydrogen bonding in juxtaposing and stacking of phenyl rings in the benzyl group side chains. Pi stacking is shown with cyan dotted lines, and the hydrogen bonding is shown in yellow dotted lines. 


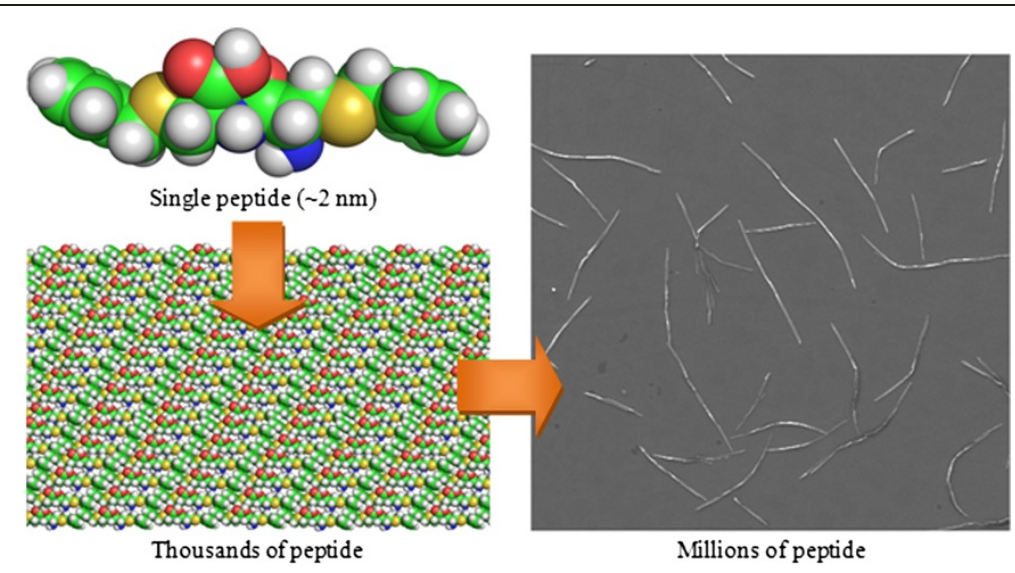

Figure 4 Schematic presentation of nanotube formation using cystine dipeptide as template. The peptide is about $2 \mathrm{~nm}$ long; tens and hundreds of thousands of individual peptides self-assemble into nanotubes.

\section{Conclusion}

This study clearly demonstrates that short water-soluble benzylcystine dipeptide self-assembles to form a supramolecular extended structure with $\beta$-sheet-like intermolecular hydrogen-bonding pattern in solid state, and this dipeptide also forms an ordered nanostructure from an aqueous solution under the proper conditions. The molecular arrangement of the self-association of this fibrilforming peptide is explained using molecular modelling studies. This work may help guide the design of biomaterial scaffoldings in thefuture by inscribing biological signals in the self-assemblies. Moreover, biocompatibility of peptide nanotubes makes them valuable for applications as biosensors and in tissue engineering. The field of nanoscale device development based on peptide nanotubes is still very nascent. Our efforts had continually been in developing such exotic nanomaterials for further advancement of this field.

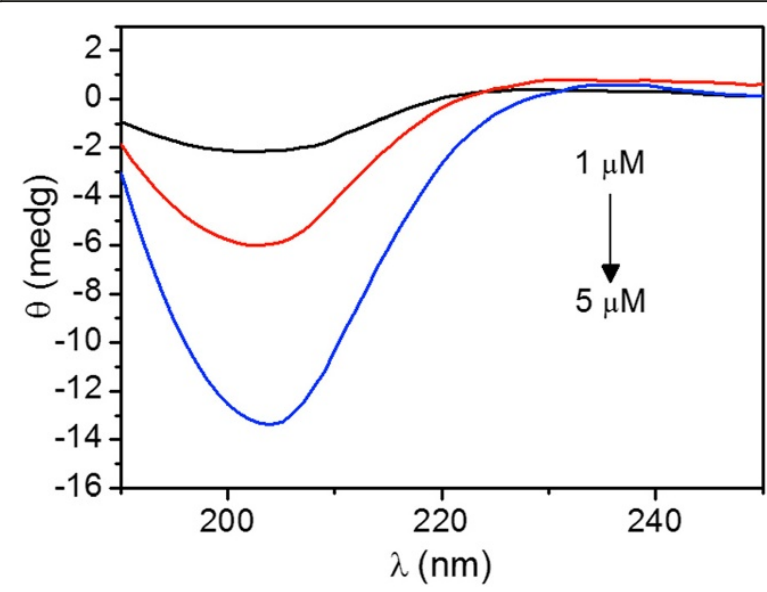

Figure $\mathbf{5}$ Circular dichroism spectra of the dipeptide at different dilutions. Approximately 3-nm shift in the CD band was observed.

\section{Methods}

\section{Synthesis of the dipeptide}

The starting materials, Boc-S-benzyl-L-cysteine, were purchased from Across Organics, and S-benzyl-L-cysteine was purchased from Alfa Aesar (Ward Hill, MA, USA) and were used without further purification. Solvents were freshly distilled by standard procedures prior to use. The dipeptide was purified by column chromatography. All ${ }^{1} \mathrm{H}$ and ${ }^{13} \mathrm{C}$-NMR spectra were recorded on a Bruker 600$\mathrm{MHz}$ spectrometer (Bruker AXS, Inc., Madison, MI, USA). For ${ }^{1} \mathrm{H}$ NMR, tetramethylsilane served as internal standard $(\delta=0)$, and data are reported as follows: chemical shift, integration, multiplicity ( $\mathrm{s}=$ singlet, $\mathrm{d}=$ doublet, $\mathrm{t}=$ triplet, $\mathrm{q}=$ quartet, $\mathrm{m}=$ multiplet), and coupling constant(s) in hertz. High-resolution mass spectra were obtained on a Jeol MS station 700 (JEOL Ltd., Akishima-shi, Japan).

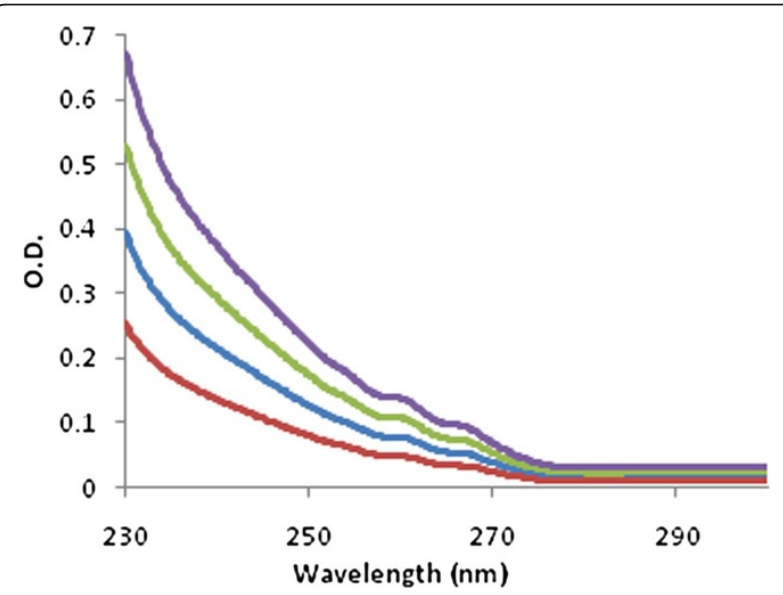

Figure 6 Absorption spectra of dipeptide at four different concentrations. Band for the benzyl groups appeared at 260 and $267 \mathrm{~nm}$. Scattering was observed due to aggregation of the peptide. OD, optical density. 


\section{FT-IR study}

The FT-IR spectra of the samples were recorded on a JASCO FT-IR 4200 spectrometer (Jasco, Easton, MD, USA) by $\mathrm{KBr}$ disc technique. The peptide was mixed with $\mathrm{KBr}$ in a clean glass pestle and compressed to obtain a pellet. The spectra were recorded from 400 to $4,000 \mathrm{~cm}^{-1}$. Background spectra were obtained with $\mathrm{KBr}$ pellet for each sample. JASCO software was used for data processing.

\section{SEM imaging}

The dipeptide sample slides were dried completely under a critical point dryer (Quorum Technologies Inc., Guelph, Canada), and after drying, the samples were kept on the sample holder using carbon tape. Gold coating was done with the current of $10 \mathrm{~mA}$ at $10-6$ to 10 $8 \mathrm{mbar} / \mathrm{Pa}$ (model no. SC7620, Quorum Technologies Inc.). SEM imaging was performed using TESCAN Vega II LSU (TESCAN Digital Microscopy Imaging, TESCAN, Brno, Czech Republic). Imaging and measurements were done using Vega TC software (TC Software, Hampton, VA, USA).

\section{AFM imaging}

Twenty microliters of the dipeptide sample $(5 \mathrm{mg}$ dissolved in $1 \mathrm{~mL}$ water) was deposited onto freshly cleaved muscovite Ruby mica sheet (ASTM V1 Grade Ruby Mica, Micafab India Pvt. Ltd., Chennai, India) for 5 to $10 \mathrm{~min}$. Mica sheets are basically negatively charged, so the dipeptide molecule binds strongly on the mica surface. After $10 \mathrm{~min}$, the sample was dried using a vacuum dryer. AAC-mode atomic force microscopy was performed using a Pico Plus 5500 AFM (Agilent Technologies, Inc., Santa Clara, CA, USA) with a piezo scanner maximum range of $9 \mu \mathrm{m}$. Microfabricated silicon cantilevers of $225 \mu \mathrm{m}$ in length with a nominal spring force constant of 21 to $98 \mathrm{~N} / \mathrm{m}$ were used from nanosensors. Cantilever oscillation frequency was tuned into resonance frequency. The cantilever resonance frequency was 150 to $300 \mathrm{kHz}$. The images $(512 \times 512$ pixels $)$ were captured with a scan size between 0.5 and $5 \mu \mathrm{m}$ at the scan speed rate of $0.5 \mathrm{rpm}$. The images were processed by flattening using Pico view software (Molecular Imaging Inc., Ann Arbor, MI, USA). The image presented in this paper was derived from the original data. Length, height, and width were measured manually using Pico view software.

\section{CD spectroscopy}

CD spectra (190 to $400 \mathrm{~nm}$ ) were acquired at $200-\mathrm{nm} / \mathrm{min}$ scan speed, with 1-nm bandwidth on a Jasco J-810 spectrometer using a 1-mm-path-length quartz cell. Peptide stock solutions $(2 \mathrm{mg} / \mathrm{mL})$ were prepared in water and appropriately diluted during $\mathrm{CD}$ measurements. Five spectra were averaged to improve the signal-to-noise ratio and smoothed using the noise-reducing option. The results were represented as machine unit $(\theta$, in units of mdeg).

\section{UV spectroscopy}

Absorption spectra were acquired using a Shimadzu UV-2401PC spectrophotometer (Shimadzu Corporation, Kyoto, Japan) with 10-mm-path-length quartz cuvette. A very dilute concentration of the peptide was used (5 to $20 \mu \mathrm{M})$.

\section{DFT calculations}

Structure of the dipeptides was drawn on Schrodinger Maestro molecular modeling environment (http://www. schrodinger.com/). Geometry was optimized using simulated annealing algorithm with the help of Desmond molecular dynamics simulations software tool. Simulation was run for $5.2 \mathrm{~ns}$ in SPC water environment in an orthorhombic periodic boundary condition. The model system was relaxed before simulation. Number of particle $(N)$, and volume of system $(V)$ in the ensemble were constant and the system had a well defined temperature $(T)$. The molecule was cooked at a high temperature and then slowly cooled down to $300 \mathrm{~K}$. The process was repeated several times [22]. The lowest energy conformation at $300 \mathrm{~K}$ was chosen for further analysis using DFT implemented in Gaussian 09 software (Gaussian Inc., Wallingford, CT, USA). In Gaussian 09, geometry optimizations and frequency calculations were performed in vacuo with B3LYP density functional using $6-311 \mathrm{G}+(2 \mathrm{~d}, \mathrm{p})$ basis set $[23,24]$.

\section{Competing interests}

The authors declare that they have no competing interests.

\section{Authors' contributions}

$\mathrm{BB}, \mathrm{SKP}$, and UP conceived and designed the experiments. SKP and UP performed the experiments. All the authors analyzed the data. SKP and UP drafted the manuscript. All authors read and approved the final manuscript.

\section{Authors' information}

Biswadip Banerji had his early education in the Ramakrishna Mission School \& Colleges, Rahara, West-Bengal and post-graduation studies in Pure Chemistry from Calcutta University. He joined Prof. Javed Iqbal's group at Indian Institute of Technology-Kanpur (IIT-K) to do PhD studies in the field of Bio-Organic Chemistry. Soon after his graduation, he worked in Regional Research Laboratory-Trivandrum (RRL-T) as a research associate for 1 year and thereafter joined Prof. Christopher J. Schofield's group as a postdoctoral researcher at the OCMS, Oxford University. After spending more than 3 years at Oxford, he joined Prof. K. C. Nicolaou's group at ICES-Singapore in 2006, where he was a research fellow working in the area of total synthesis of complex natural products. Soon after the completion of his postdoctoral studies, in May 2008, he returned to India and joined 'Chembiotek International' as a team-leader. He has taken up his current senior scientist position at IICB, one of the renowned research institutes under the umbrella of CSIR, from January 2009. His current research interests are broadly on Chemical biology, mainly on the structure-based drug design/enzyme inhibition focusing natural product-based privileged scaffolds. He also has great interest in the cutting edge research on nano- and biotechnology, particularly on the synthesis of 'smart molecules' and their application in therapeutics and pharmaceuticals.

Sumit Kumar Pramanik obtained his B.Sc. in Chemistry from Vidyasagar University, India. He earned his M.Sc. in Applied Chemistry from Bengal Engineering and Science University, Shibpur, India. He is a Ph.D. student from 
the Chemistry Division, Indian Institute of Chemical Biology, Kolkata, India. His research area interests include nanobiomaterials and peptide-based drug design and biophysical chemistry.

Uttam Pal earned his B.Sc. in Physiology from the Presidency College, Kolkata, India. He is a M.Sc. degree holder of Biophysics and Molecular Biology from University of Calcutta, Kolkata, India. He is a Ph.D. student from the Structural Biology and Bioinformatics Division, Indian Institute of Chemical Biology, Kolkata, India. His research area covers structural biology and bioinformatics.

Nakul Chandra Maiti achieved M.Sc. in Chemistry, University of Calcutta, Kolkata, India; Ph.D. From Tata Institute of Fundamental Research, Mumbai, India; Postdoctoral JSPS visiting scientist, Institute for Molecular Science, Japan; Postdoctoral Senior Research Associate, Biochemistry, Case, Cleveland, Ohio, USA; Postdoctoral Research Associate/lecturer, California State University, Los Angeles, USA. He is a senior scientist from the Indian Institute of Chemical Biology, Kolkata, India. His research area interests cover structure-based amyloid research, structural aspects and in vitro behavior of natively unfolded proteins and peptides those are linked to human diseases, applications of NMR, fluorescence and Raman spectroscopy to biological systems, computational biochemistry, and bioinformatics.

\section{Acknowledgements}

SP gave thanks to CSIR-India, UP thanks INSPIRE Fellowship Programme, DST, India, for financial support. We would also like to thank CSIR-IICB, India, for providing financial assistance.

\section{Author details}

'Department of Chemistry, CSIR-Indian Institute of Chemical Biology, 4, Raja S.C. Mullick Road, Kolkata 700032, India. ${ }^{2}$ Department of Structural Biology and Bioinformatics, CSIR-Indian Institute of Chemical Biology, 4, Raja S.C. Mullick Road, Kolkata 700032, India.

Received: 8 March 2013 Accepted: 27 March 2013 Published: 17 April 2013

\section{References}

1. Cui, Y, Wei, Q, Park, H, Lieber, CM: Nanowire nanosensors for highly sensitive and selective detection of biological and chemical species. Science 293(5533), 1289-1292 (2001)

2. Diehl, MR, Yaliraki, SN, Beckman, RA, Barahona, M, Heath, JR: Self-assembled, deterministic carbon nanotube wiring networks. Angew Chem Int Ed Engl 41(2), 353-356 (2002)

3. Collins, PG, Arnold, MS, Avouris, P: Engineering carbon nanotubes and nanotube circuits using electrical breakdown. Science 292(5517), 706-709 (2001)

4. Gao, X, Matsui, H: Peptide-based nanotubes and their applications in bionanotechnology. Adv. Mater. 17(17), 2037-2050 (2005)

5. Wang, Y, Dai, Q, Zou, B, Yu, WW, Liu, B, Zou, G: Facile assembly of size- and shape-tunable IV-VI nanocrystals into superlattices. Langmuir 26(24), 19129-19135 (2010)

6. Whitesides, GM: Nanoscience, nanotechnology, and chemistry. Small 1(2), 172-179 (2005)

7. Martin, CR, Kohli, P: The emerging field of nanotube biotechnology. Nat Rev Drug Discov 2, 29-37 (2003)

8. Bong, DT, Clark, TD, Granja, JR, Ghadiri, MR: Self-assembling organic nanotubes. Angew. Chem. Int. Ed. 40(6), 988-1011 (2001)

9. Gazit, E: Self-assembled peptide nanostructures: the design of molecular building blocks and their technological utilization. Chem. Soc. Rev. 36(8), 1263-1269 (2007)

10. Lv, C, Xue, Q, Shan, M, Jing, N, Ling, C, Zhou, X, Jiao, Z, Xing, W, Yan, Z: Selfassembly of double helical nanostructures inside carbon nanotubes. Nanoscale (2013). doi:10.1039/C2NR33157H

11. Colombo, G, Soto, P, Gazit, E: Peptide self-assembly at the nanoscale: a challenging target for computational and experimental biotechnology. Trends Biotechnol. 25(5), 211-218 (2007)

12. Brea, RJ, Granja, JR: Self-assembly of cyclic peptides in hydrogen-bonded nanotubes. In: Schwarz, JA, Contescu, Cl, Putyera, K (eds.) Dekker Encyclopedia of Nanoscience and Nanotechnology, 1st edn, pp. 3439-3457. Marcel Dekker, New York (2004)
13. Ghadiri, MR, Granja, JR, Milligan, RA, MCRee, DE, Khazanovich, N: Selfassembling organic nanotubes based on a cyclic peptide architecture. Nature 366(6453), 324-327 (1993)

14. Khazanovich, N, Granja, JR, Milligan, RA, McRee, DE, Ghadiri, MR: Nanoscale tubular ensembles with specified internal diameters. Design of a selfassembled nanotube with a $13 \AA$ pore. J. Am. Chem. Soc. 116(13), 6011-6012 (1994)

15. Ghadiri, MR, Granja, JR, Buehler, LK: Artificial transmembrane ion channels from self-assembling peptide nanotubes. Nature 369(6478), 301-304 (1994)

16. Panciera, M, Amorín, M, Castedo, L, Granja, JR: Design of stable $\beta$-sheet -based cyclic peptide assemblies assisted by metal coordination: selective homo- and heterodimer formation. Chemistry (2013). doi:10.1002/ chem.201203213

17. Amin, R, Kim, S, Park, SH, LaBean, T: Artificially designed DNA nanostructures. NANO: Brief Reports and Reviews 4(3), 119-139 (2009)

18. Maity, S, Jana, P, Maity, SK, Haldar, D: Fabrication of hollow self-assembled peptide microvesicles and transition from sphere-to-rod structure. Langmuir 27(7), 3835-3841 (2011)

19. Tao, K, Wang, J, Zhou, P, Wang, C, Xu, H, Zhao, X, Lu, JR: Self-assembly of short $A \beta(16-22)$ peptides: effect of terminal capping and the role of electrostatic interaction. Langmuir 27(6), 2723-2730 (2011)

20. Tian, F, Cziferszky, M, Jiao, D, Wahlströ m, K, Geng, J, Scherman, OA: Peptide separation through a $\mathrm{CB}[8]$-mediated supramolecular trap-and-release process. Langmuir 27(4), 1387-1390 (2010)

21. Hauser, CAE, Zhang, S: Designer self-assembling peptide nanofiber biological materials. Chem. Soc. Rev. 39(8), 2780-2790 (2010)

22. Banerji, B, Pramanik, SK, Pal, U, Maiti, NC: Conformation and cytotoxicity of a tetrapeptide constellated with alternative $\mathrm{d}$ - and I-proline. RSC Advances 2(17), 6744-6747 (2012)

23. Banerji, B, Pramanik, SK, Mandal, S, Maiti, NC, Chaudhuri, K: Synthesis, characterization and cytotoxicity study of magnetic $\left(\mathrm{Fe}_{3} \mathrm{O}_{4}\right)$ nanoparticles and their drug conjugate. RSC Advances 2(6), 2493-2497 (2012)

24. Rudra, DS, Pal, U, Maiti, NC, Reiter, RJ, Swarnakar, S: Melatonin inhibits matrix metalloproteinase-9 activity by binding to its active site. J Pineal Res (2012). doi:10.1111/jpi.12034

doi:10.1186/2193-8865-3-12

Cite this article as: Banerji et al:: Dipeptide derived from benzylcystine forms unbranched nanotubes in aqueous solution. Journal of Nanostructure in Chemistry 2013 3:12.

\section{Submit your manuscript to a SpringerOpen ${ }^{\odot}$ journal and benefit from:}

- Convenient online submission

$\checkmark$ Rigorous peer review

- Immediate publication on acceptance

- Open access: articles freely available online

- High visibility within the field

- Retaining the copyright to your article

Submit your next manuscript at $>$ springeropen.com 